\title{
PERCEPÇÃO DO PACIENTE RENAL CRÔNICO ACERCA DOS CUIDADOS COM ACESSOS PARA HEMODIÁLISE
}

\author{
Flávia Lidyane Lima Nogueira ${ }^{1}$, Larissa Rodrigues de Freitas ${ }^{2}$, Nivia da Silva Cavalcante ${ }^{3}$, Viviane Peixoto dos \\ Santos Pennafort ${ }^{4}$
}

RESUMO: Objetivou-se descrever os cuidados do paciente renal crônico com seu acesso para hemodiálise e relatar sobre as orientações e cuidados recebidos pela equipe de enfermagem. Estudo descritivo, desenvolvido em uma clínica de hemodiálise localizada em Fortaleza, estado do Ceará, no período de outubro e novembro de 2014, com 28 pacientes em tratamento hemodialítico. Utilizou-se entrevista semiestruturada para coleta de dados. Optou-se pela análise temática, sendo elencadas as categorias: Cuidado com o acesso e suas implicações no cuidado de si, e Percepção do paciente renal crônico acerca das orientações e cuidados recebidos da equipe de enfermagem. Os pacientes destacaram as dúvidas relacionadas às mudanças de hábitos e a preocupação da enfermagem na perviedade dos acessos, com orientações quanto aos cuidados domiciliares e as intervenções no serviço de diálise. Percebe-se a necessidade de estratégias educativas junto ao paciente na aquisição de novas habilidades para a preservação do acesso para hemodiálise.

DESCRITORES: Fístula Arteriovenosa; Cateteres Venosos Centrais; Cuidados de Enfermagem; Diálise renal; Insuficiência renal crônica.

\section{PERCEPTION OF PATIENTS WITH CHRONIC KIDNEY DISEASE REGARDING CARE TOWARDS THEIR HEMODIALYSIS ACCESS}

ABSTRACT: The aim of this study was to describe the care of patients with chronic kidney disease towards their hemodialysis access, and report on the guidelines and care given by the nursing staff. This is a descriptive study, developed in a hemodialysis clinic located in the city of Fortaleza, state of Ceará, between October and November 2014, with 28 patients on hemodialysis. Semi-structured interviews were undertaken to collect data. The thematic analysis technique was chosen, with the following categories: Care towards the hemodialysis access and its implications in self-care, and Perception of patients with chronic kidney disease on guidance and care received from the nursing staff. Patients highlighted the doubts related to changes in habits, and concerns of the nursing staff about patency of access, with guidance on home care and interventions in the dialysis service. Educational strategies to the patient in the acquisition of new skills for the preservation of hemodialysis access are necessary.

DESCRIPTORS: Arteriovenous Fistula; Central Venous Catheters; Nursing Care; Renal Dialysis; Chronic Renal Insufficiency.

\section{PERCEPCIÓN DEL PACIENTE RENAL CRÓNICO RESPECTO AL CUIDADO DE SU ACCESIBILIDAD A LA HEMODIÁLISIS}

\begin{abstract}
RESUMEN: Se objetivó describir el cuidado del paciente renal crónico respecto de su acceso a hemodiálisis e informar sobre indicaciones y cuidados recibidos del equipo de enfermería. Estudio descriptivo, desarrollado en clínica de hemodiálisis en Fortaleza, estado de Ceará, entre octubre y noviembre de 2014, con 28 pacientes en tratamiento de hemodiálisis. Datos recolectados mediante entrevista semiestructurada. Se eligió el análisis temático, surgiendo las categorías: Cuidado de la accesibilidad e implicancias en el cuidado en sí; y Percepción del paciente renal crónico respecto de indicaciones y cuidados recibidos del equipo de enfermería Los pacientes destacaron dudas relacionadas a cambios de hábitos y a la preocupación de enfermería por la permeabilidad de los accesos, con indicaciones referentes a cuidados domiciliarios y a intervenciones en servicio de diálisis. Se observa necesidad de estrategias educativas con el paciente para la adquisición de nuevas habilidades de preservación de la accesibilidad a la hemodiálisis. DESCRIPTORES: Fístula Arteriovenosa; Catéteres Venosos Centrales; Atención de Enfermería; Diálisis Renal; Insuficiencia Renal Crónica.
\end{abstract}

${ }^{1}$ Enfermeira. Enfermeira da Estratégia de Saúde da Família Maria Aparecida Pinheiro. Distrito de Betânia, CE, Brasil.

${ }^{2}$ Enfermeira. Preceptora de estágio do Curso Técnico em Enfermagem da instituição Elite. Fortaleza, CE, Brasil.

${ }^{3}$ Enfermeira. Enfermeira do Centro de Atenção Psicossocial Álcool e Drogas. Maracanaú, CE, Brasil.

${ }^{4}$ Enfermeira. Doutora em Enfermagem. Docente de Enfermagem do Centro Universitário Estácio do Ceará. Fortaleza, CE, Brasil.

Autor Correspondente:

Viviane Peixoto dos Santos Pennafort

Centro Universitário Estácio do Ceará

R. Eliseu Uchôa Beco, 600 - 60810-270 - Fortaleza, CE, Brasil

E-mail: vivipspf@yahoo.com.br
Recebido: 01/03/2016

Finalizado: 08/09/2016 


\section{INTRODUÇÃO}

O cuidado do paciente renal crônico em tratamento hemodialítico envolve o incentivo ao autocuidado, prevenção de infecções, fornecimento de informações ao paciente e família em relação ao seu tratamento e complicações, como também prover um local segu ro e confortável para a realização do tratamento ${ }^{(1)}$.

Os distúrbios renais podem se apresentar de forma aguda ou crônica. A insuficiência renal aguda (IRA) se dá por alguma lesão no rim. Neste caso, o tratamento consiste na substituição da função renal temporariamente para diminuir as prováveis complicações e reduzir os riscos de aumentar a lesão. A doença renal crônica (DRC) é também causada por uma lesão renal, acompanhada de uma diminuição da taxa de filtração glomerular (TFG) ${ }^{(2)}$.

Em 2013, o número de unidades de diálise ativas no Brasil era de 658 unidades, com total estimado de 100.397 pacientes por ano, desses, 84\% dialisavam pelo Sistema Único de Saúde (SUS) e 16\% por outros convênios. Quanto à modalidade dialítica, $90 \%$ dos pacientes estavam em tratamento hemodialítico e apenas $10 \%$ em diálise peritoneal. A faixa etária de 19 a 64 anos apresentou maior incidência. E o sexo predominante foi o masculino com $58 \%{ }^{(3)}$.

Quando o rim diminui ou perde sua capacidade de exercer sua função normal, é necessário usar métodos substitutivos para manter-se vivo e com qualidade de vida, como a diálise peritoneal (DP), hemodiálise (HD) ou transplante renal. Desses, o mais utilizado é a hemodiálise, que consiste em remover os solutos acumulados no sangue como a creatinina, ureia, potássio, fosfato e água, realizando a reposição parcial da função desempenhada pelos rins ${ }^{(2)}$.

Para realizar a hemodiálise, o paciente necessita de um acesso vascular como uma fístula arteriovenosa (FAV), cateter venoso de duplo lúmen (CDL) ou enxerto. A FAV se tornou para o paciente em tratamento dialítico um meio importante para a realização do tratamento, considerada um método seguro que proporciona conforto e autonomia em relação ao $\mathrm{CDL}^{(4)}$.

A equipe de enfermagem está diretamente envolvida na assistência ao paciente em tratamento hemodialítico e o enfermeiro deve incentivar o desenvolvimento de sua capacidade de autocuidado por meio do conhecimento, o que norteará o paciente na aquisição de habilidades para atuar em situações de complicações com seu acesso vascular ${ }^{(5)}$.

Nessa perspectiva, verificou-se que não adianta somente a equipe multiprofissional ter cuidado com esse paciente e seus acessos vasculares, mas o próprio paciente necessita ser orientado e saber de suas contribuições em relação à sua manutenção. E esses cuidados devem ser realizados tanto na unidade de tratamento quanto no ambiente domiciliar, estimulando esses pacientes ao cuidado de si.

Considera-se que o autocuidado deve ser construído de maneira conjunta entre profissionais e pacientes, a partir de uma prioridade escolhida ${ }^{(6)}$.

A qualidade de vida do paciente pode ser melhorada ao encorajar o autocuidado. Dessa forma, a equipe de enfermagem deve fornecer apoio e capacitar o paciente, por meio de educação continuada informando sobre sua doença, limitações, sinais e sintomas e principalmente os cuidados com o acesso para a diálise ${ }^{(4)}$.

Ante a complexidade da insuficiência renal crônica, o paciente renal necessita de orientações da equipe de enfermagem para atuar no cuidado de si, pois geralmente demonstram alguma dificuldade na realização das atividades cotidianas inerentes ao tratamento ${ }^{(7)}$.

Neste contexto, o presente estudo teve como objetivo descrever os cuidados do paciente renal crônico com seu acesso para hemodiálise e relatar sobre as orientações e cuidados recebidos da equipe de enfermagem.

\section{MÉTODO}


Estudo descritivo com abordagem qualitativa, realizado no período de outubro e novembro de 2014 em uma clínica de diálise do município de Fortaleza, que atende atualmente 164 pacientes da rede particular e convênios. Recebe mensalmente uma média de 10 novos pacientes. Tem uma estrutura de duas salas para hemodiálise, uma com 32 máquinas e outra com quatro máquinas. Tem serviço de diálise peritoneal. Trabalha com uma equipe de cinco enfermeiros, 19 técnicos de enfermagem, um psicólogo, um assistente social, um nutricionista e quatro médicos.

Os participantes foram pacientes portadores de DRC em tratamento hemodialítico que atenderam aos seguintes critérios de inclusão: maiores de 18 anos, em hemodiálise, que utilizavam cateter, ou FAV no período máximo de 06 meses. O critério de exclusão foi: pacientes sem condições clínicas no período da coleta.

A coleta de dados ocorreu por entrevista semiestruturada, gravadas com auxílio de um gravador. com as seguintes questões: Como você realiza os cuidados do acesso venoso (cateter ou fístula)? Quais as orientações que você recebeu no serviço?

Optou-se pela análise temática ${ }^{(8)}$, que se desenvolve nas seguintes etapas: transcrição na íntegra das entrevistas, pré-análise com leitura flutuante, síntese do conteúdo e leitura exploratória. Os dados foram agrupados em conjuntos e subconjuntos que demonstravam as mesmas ideias e posteriormente foram reclassificados com base na relevância do presente estudo. Dessa forma, emergiram duas categorias: I - Cuidado com o acesso e suas implicações no cuidado de si; e II - Percepção do paciente renal crônico acerca das orientações e cuidados recebidos da equipe de enfermagem.

Inicialmente o projeto de pesquisa foi apresentado à direção da instituição e à gerência de enfermagem da unidade de hemodiálise, para autorização da coleta de dados junto aos pacientes e permissão de acesso aos prontuários, por meio da Carta de Anuência e Termo de Fiel Depositário, respectivamente. O trabalho foi submetido ao Comitê de Ética em Pesquisa da Universidade Estadual do Ceará - UECE, com parecer de $\mathrm{n}^{\circ} 229.772$. O conteúdo gravado foi transcrito, resguardando o sigilo e anonimato dos pacientes substituindo seus nomes por nomes de pedras preciosas.

\section{RESULTADOS}

Foram entrevistados 28 pacientes. Conforme categorização no Quadro1, observa-se a predominância do sexo feminino, a faixa etária prevalente foi de 41 a 60 anos. Do total, 15 (53,6\%) eram casados, com uma renda média maior ou igual a dois salários mínimos. As principais comorbidades associadas à doença renal crônica foram a hipertensão arterial sistêmica e o diabetes mellitus. Em relação ao acesso venoso, $15(53,6 \%)$ dos pacientes estavam em uso de algum tipo de cateter venoso central, sendo ele de curta ou longa permanência e $13(46,4 \%)$ estavam em uso de FAV. Observou-se que dentre os pacientes, 19 (67,8\%) iniciaram o tratamento dialítico em outras clínicas.

A partir da análise dos relatos, duas categorias foram elencadas: I - Cuidado com o acesso e suas implicações no cuidado de si; II - Percepção do paciente renal crônico acerca das orientações e cuidados recebidos da equipe de enfermagem.

\section{Cuidado com o acesso e suas implicações no cuidado de si}

Nos depoimentos coletados, verifica-se uma preocupação maior com a permanência e manutenção do acesso, as quais demandam muitos cuidados domiciliares, com modificação significativa dos hábitos de vida. O uso do cateter venoso central para hemodiálise causa-lhes maior desconforto quando comparado à fístula arteriovenosa, como pode ser observado nos relatos a seguir:

[...] Eu não estou mais fazendo nada, estou parado, para não machucar [pescoço]. Dificultou muito o banho. (Fuxita)

Eu procuro não molhar o cateter. Nunca molho, eu sempre coloco uma toalhinha, em cima, ou coloco um filme, e depois passo o micropore do lado pra tentar tomar banho tranquila, mas não consigo porque não posso molhar. A única forma que tenho é tentar prevenir o máximo. (Diamante) 
Agora no estado que me encontro eu só lavo bem ela [FAV] com asseptol, e uso sempre band aid. Todo dia eu troco. Duas vezes por dia. (Safira)

Contudo, observou-se que cada paciente adapta as rotinas do seu dia a dia na presença do acesso.

[...] eu não durmo do lado dela [FAV], sempre deixo protegendo, faço exercício com a bolinha, aí quando tem hematoma, passo gelo e água quente. (Cornalina)

Eu tenho maior cuidado pra não pegar peso, eu asseio meu braço bem asseado [braço da FAV]. Quando é de noite eu boto uma compressa de gelo, ou então pano quente [...]. Quando vou tomar banho tenho o sabonete próprio, só dele. (Pirita)

[...] só faço mesmo amarrar uma tira aqui na minha cabeça para ele [cateter] não ficar balançando muito, coloco um boné e pronto. (Fuxita)

\section{Percepção do paciente renal crônico acerca das orientações e cuidados recebidos da equipe de enfermagem}

Percebe-se nos relatos dos pacientes que as principais orientações recebidas pela equipe de enfermagem nos cuidados com o cateter venoso central foram: evitar molhar e manipular o acesso venoso central.

[...] eles [equipe de enfermagem] pedem pra gente não mexer, fazer de tudo pra não molhar! Não manipular, eles preferem manipular aqui [clínica]. Então a gente nem mexe nele [cateter], que é exatamente para não ter contaminação, de nada. (Esmeralda)

Ele [enfermeiro] disse que quando eu for tomar banho não é para molhar, para não dar infecção. (Rubi)

Eles dizem pra gente não deixar ninguém mexer, não pegar muita poeira, [...] para evitar qualquer tipo de contaminação. (Turmalina)

Quanto aos cuidados com a FAV, os pacientes destacaram as seguintes orientações: evitar pegar peso e dormir por cima do braço ou verificar glicemia no membro com FAV, realizar exercício e higienizar o braço da FAV.

[...] não dormir em cima do braço, não pegar peso, não botar nada assim, minha diabete não furar mais no braço [não aplicar insulina], não medir a pressão, tudo. (Pirita)
Tabela 1 - Caracterização dos pacientes renais crônicos em tratamento hemodialítico, Fortaleza, CE, Brasil, 2014

\begin{tabular}{|c|c|c|}
\hline Características & $\mathbf{N}$ & $\%$ \\
\hline \multicolumn{3}{|l|}{ Sexo } \\
\hline Masculino & 11 & 39 \\
\hline Feminino & 17 & 61 \\
\hline \multicolumn{3}{|l|}{ Idade (em anos) } \\
\hline $20-40$ & 8 & 28,6 \\
\hline $41-60$ & 13 & 46,4 \\
\hline$>60$ & 7 & 25 \\
\hline \multicolumn{3}{|l|}{ Estado civil } \\
\hline Solteiro(a) & 6 & 21,4 \\
\hline Casado(a) & 15 & 53,6 \\
\hline Divorciado(a) & 3 & 10,8 \\
\hline Viúvo(a) & 4 & 14,2 \\
\hline \multicolumn{3}{|l|}{ Escolaridade } \\
\hline Fundamental Completo & 4 & 14,3 \\
\hline Fundamental Incompleto & 8 & 28,6 \\
\hline Médio completo & 8 & 28,6 \\
\hline Médio incompleto & 3 & 10,7 \\
\hline Superior completo & 2 & 7,1 \\
\hline Superior incompleto & 3 & 10,7 \\
\hline \multicolumn{3}{|c|}{ Renda familiar (em salário mínimo) } \\
\hline$\leq 1$ & 10 & 35,8 \\
\hline$\geq 2$ & 16 & 57,1 \\
\hline Não Sabe & 2 & 7,1 \\
\hline \multicolumn{3}{|l|}{ Comorbidades } \\
\hline HAS & 9 & 32,1 \\
\hline $\mathrm{DM}$ & 1 & 3,7 \\
\hline $\mathrm{HAS}+\mathrm{DM}$ & 10 & 35,7 \\
\hline HAS + LUPUS & 2 & 7,1 \\
\hline Cardiopatias & 3 & 10,7 \\
\hline Nenhuma & 3 & 10,7 \\
\hline \multicolumn{3}{|l|}{ Acesso atual } \\
\hline Cateter Permcath & 7 & 25 \\
\hline Curta Permanência & 8 & 28,6 \\
\hline FAV & 13 & 46,4 \\
\hline \multicolumn{3}{|l|}{ Relato de infecção } \\
\hline Peritonite & 1 & 3,6 \\
\hline Infecção de Cateter & 1 & 3,6 \\
\hline Infecção de FAV & 2 & 7,1 \\
\hline \multicolumn{3}{|c|}{ Instituição de início do tratamento } \\
\hline Hospitais e outras clínicas & 19 & 67,8 \\
\hline Própria clínica & 8 & 28,6 \\
\hline Não sabe & 1 & 3,6 \\
\hline
\end{tabular}


Os cuidados necessários de lavar e de fazer aquele exercício com a bolinha, ficar sempre fazendo. (Bronzita)

Os pacientes mencionaram ainda os cuidados na manutenção do cateter venoso central realizados pela equipe de enfermagem do serviço de diálise. Destacou-se a preocupação da equipe com a manutenção do acesso e a prevenção de infecção.

Porque aqui quando a gente chega, ele [enfermeiro] limpa bem, limpa para não dar infecção [...] e não ter febre [...]. É um cuidado muito grande que eles [profissionais] têm com a gente aqui. (Rubi)

É feito o curativo na clínica. (Turmalina)

O cateter também é feito o curativo aqui. Só aqui! Eles [enfermeiros] não querem nem que a gente chegue perto [...]. (Hematita)

De acordo com os relatos dos pacientes, as principais intervenções realizadas pelas técnicas de enfermagem na manutenção da fístula arteriovenosa foram a antissepsia antes da punção e a hemostasia no final da sessão de hemodiálise.

[...] mesmo quando vai colocar a agulha elas [técnicas de enfermagem] higienizam o braço, mesmo estando lavado. [...] eles colocam tipo um band aid para estancar o sangue. (Aragonita)

Contraditoriamente, alguns relatos destacaram a falta de orientação relacionada aos cuidados com os acessos venosos.

Não, também não recebi. (Cornalina)

Não. Parece que é não molhar. (Selenita)

Não, a única orientação que recebi foi da minha vascular. (Diamante)

Destacou-se a preferência de uma paciente pela utilização do cateter de longa permanência (Permcath) à fístula, devido à inabilidade técnica dos profissionais no momento da punção da fístula, com consequente perda do funcionamento da mesma, conforme relato:

Prefiro usar o cateter no lugar da fístula porque não adianta confeccionar uma fístula se não tem profissional qualificado para puncionar, já perdi duas fístulas, eles [equipe de enfermagem] erraram na hora de colocar as agulhas. (Diamante)

\section{DISCUSSÃO}

Observou-se no período de coleta que existe um vínculo forte entre a equipe de enfermagem e os pacientes, cada profissional conhece as particularidades de cada um, trata-os pelo nome e as famílias demonstram confiança na equipe.

$\mathrm{Na}$ primeira categoria, os pacientes renais crônicos em tratamento hemodialítico mostraram-se preocupados com a manutenção do acesso, repercutindo em mudanças de hábitos e dos cuidados de si, como o banho e a maneira de dormir, a fim de evitar complicações no cateter venoso central, ou na fístula arteriovenosa.

Considera-se que o cuidado de si emerge a partir do conhecimento e aceitação de sua atual condição, com novos hábitos, possibilitando a manutenção do tratamento por meio do cuidado com o acesso e outras mudanças no seu cotidiano ${ }^{(9)}$.

Para que o cuidado de si seja incorporado no contexto de vida do paciente renal crônico, é imprescindível estimular suas capacidades, habilidades e potencial de reação humana, propiciando que ele se adapte de maneira positiva ao novo estilo de vida e assuma o controle de seu tratamento. Essa adaptação pode ser facilitada pelo enfermeiro que, por meio do cuidado, planeja intervenções educativas, visando ajudá-los a reaprender a viver com a nova realidade e a sobreviver com a doença renal crônica ${ }^{(10)}$. 
Observou-se na segunda categoria a percepção dos pacientes quanto às orientações em relação aos cuidados com o acesso para hemodiálise. Ressalta-se que os relatos enfatizaram principalmente a perviedade do acesso venoso central e manutenção do funcionamento da FAV, com vistas à continuidade do tratamento hemodialítico para manterem-se vivos, já que o funcionamento inadequado do acesso implica diretamente na interrupção do procedimento trazendo consequências fatais.

Conhecer o funcionamento da hemodiálise é fundamental no processo de adaptação do paciente às restrições, consequentemente melhorando sua contribuição com o tratamento. Com isso, o enfermeiro, além de assistir no procedimento, deve atuar como educador, facilitando o desenvolvimento do paciente com cuidado de si no seu cotidiano ${ }^{(10)}$.

Ressalta-se que, para realizar a hemodiálise, a fístula é considerada o acesso mais seguro quando se relaciona à infecção, pois sua incidência é pequena. Neste contexto, a indicação do cateter deve ser somente em casos de hemodiálise de urgência, ou enquanto uma fístula matura(11).

Percebeu-se que os pacientes têm preferência pela fístula, por melhorar a qualidade de vida ao proporcionar maior comodidade e segurança no tratamento hemodialítico. Já o cateter, apesar de não precisar puncionar toda vez que vai realizar a hemodiálise, requer cuidados mais específicos e deve-se ter atenção com higiene e curativo, além de ser um dispositivo incômodo e esteticamente desagradável.

Contraditoriamente, uma paciente ressaltou a preferência pela utilização do cateter à fístula arteriovenosa, informou que foram confeccionadas duas fístulas, que devido à punção inadequada perdeu as fístulas e estava em uso de cateter de longa permanência. Infere-se, a partir desse relato, certa insegurança acerca da falta de habilidade de alguns profissionais no momento da punção da fístula arteriovenosa.

Destarte, a especificidade e complexidade da atuação da equipe de enfermagem junto aos procedimentos dialíticos; a fragilidade do paciente renal crônico; a importância da manutenção e uso competente do acesso vascular, é de responsabilidade do enfermeiro ou técnico de enfermagem a realização da punção da fístula arteriovenosa, desde que devidamente capacitado e no caso do técnico de enfermagem, sob orientação e supervisão de enfermeiro. Ressalta-se que a primeira punção da fístula é de competência exclusiva do enfermeiro ${ }^{(12)}$.

Considera-se que a função do enfermeiro é promover o bem estar do paciente renal em tratamento hemodialítico, dentro dos seus limites e respeitando a opção de cada um, ressaltando que cada individuo é responsável pelo sucesso do seu tratamento ${ }^{(11)}$.

Os participantes relataram ainda, na segunda categoria, como é realizado o cuidado dos profissionais no serviço. Expressaram a preocupação dos profissionais principalmente em relação à higiene e manutenção dos acessos, com ênfase na prevenção de infecções.

A infecção de cateter está associada à falta de técnicas assépticas e manuseio inadequados desses dispositivos, corroborando com o presente estudo ${ }^{(13)}$. Para evitar danos à saúde do paciente renal em tratamento hemodialítico, a equipe de enfermagem necessita manter-se capacitada para atuar na promoção e prevenção de complicações, proporcionando maior confiança do paciente em relação à equipe ${ }^{(14)}$.

O estudo mostrou que as situações vivenciadas pelos pacientes nos cuidados domiciliares com o cateter venoso central ou com a fístula arteriovenosa são singulares e que a falta de informação acarreta sérios prejuízos na preservação do acesso para hemodiálise, o que requer assistência multidisciplinar individualizada, com orientações contínuas em busca da autonomia no cuidado de si.

É notório nesse contexto, que o papel do enfermeiro-educador deve ser o de negociar o cuidado, valorizar os saberes, práticas cuidativas e crenças do paciente renal crônico na reestruturação da vida e no enfrentamento dos desafios cotidianos. Nesta perspectiva, há necessidade de maior envolvimento da equipe na promoção da educação continuada dos pacientes com ênfase nos cuidados para a manutenção da via de tratamento e na prevenção de infecção, favorecendo um tratamento hemodialítico eficaz, melhorando assim a qualidade de vida. 
Nas categorias descritas, observou-se que o paciente renal crônico apresenta um conhecimento prévio acerca dos cuidados com os acessos, no entanto, eles se mostram presos a um único discurso: não molhar o cateter e não dormir ou pegar peso do lado do braço da fístula.

Com relação à atuação da equipe de enfermagem, os relatos destacaram que alguns pacientes não foram orientados adequadamente quanto aos cuidados com o acesso para hemodiálise, por outro lado, alguns enfatizaram a preocupação dos profissionais em relação à manutenção e prevenção de complicações.

Portanto, deve-se considerar a percepção que cada paciente com insuficiência renal crônica tem das orientações recebidas para o cuidado domiciliar na manutenção do acesso para hemodiálise. Ao valorizar suas sugestões e dúvidas nos cuidados diários, o enfermeiro desenvolverá estratégias criativas nas salas de hemodiálise capazes de facilitar a comunicação e a compreensão dos assuntos abordados.

Os achados desta pesquisa são restritos à instituição em que foi realizada e com um número reduzido de participantes, no entanto, apresentaram semelhança com outros serviços. Dessa forma, percebe-se que a temática necessita de outras investigações, a fim de apreender o conhecimento de outros pacientes no cuidado domiciliar com o acesso para hemodiálise. A partir dessa abordagem, será possível desenvolver cursos de capacitação e atualização para os profissionais envolvidos no planejamento e execução das ações educativas, junto aos pacientes renais crônicos e familiarescuidadores.

\section{- REFERÊNCIAS}

1. Frazão CMFQ, Delgado MFD, Araújo MGA, Silva FBBL, Sá JD, Lira ALBC. Cuidados de enfermagem ao paciente renal crônico em hemodiálise. Rev Rene. 2014; 15(4): 701-9.

2. Riella MC. Princípios de Nefrologia e Distúrbios Hidroeletrolíticos. $5^{\text {a }}$ ed. Rio de Janeiro: Guanabara Koogan; 2011.

3. Sociedade Brasileira de Nefrologia (SBN). Censo Brasileiro de Diálise [Internet] 2013 [acesso em 23 abr 2014]. Disponível: http://www.sbn.org.br/censo-brasileiro.

4. Maniva SJCF, de Freitas CHA. O paciente em hemodiálise: autocuidado com a fístula arteriovenosa. Rev Rene. 2010; 11(1): 152-60.

5. Sousa CN. Cuidar da pessoa com fístula arteriovenosa: modelo para a melhoria contínua. Rev Port Saúde Pública. 2012; 30(1): 11-7.

6. Ministério da Saúde (BR). Secretaria de Atenção à Saúde. Departamento de Atenção Especializada e Temática. Diretrizes clínicas para o cuidado ao paciente com doença renal crônica - DRC no Sistema Único de Saúde. Brasília: Ministério da Saúde; 2014.

7. de Ferreira AFA. O papel do enfermeiro na assistência de enfermagem ao paciente em tratamento hemodialítico (Revisão de Literatura) [monografia]. Recife: Instituto Nacional de Ensino e Pesquisa; 2014.

8. Minayo MCS. O Desafio do Conhecimento. 10ª Ed. São Paulo: Hucitec; 2007.

9. Roso CC, Beuter M, Kruse MHL, Girardon-Perlini NMO, Jacobi CS, Cordeiro FR. O cuidado de si de pessoas em tratamento conservador da insuficiência renal crônica. Texto Contexto Enferm. 2013;22(3):739-45.

10. dos Santos I, Rocha RPF, Berardinelli LMM. Necessidades de orientação de enfermagem para o autocuidado de clientes em terapia de hemodiálise. Rev. bras. enferm. 2011; 64(2): 335-42.

11. das Neves Júnior MA, Petnys A, Melo RC, Rabboni E. Acesso Vascular para Hemodiálise: O que há de novo. J Vasc Bras. 2013; 12(3): 221-5. 
12. Conselho Regional de Enfermagem de São Paulo (COREN - SP). Parecer n. 042, de 29 de maio de 2013. Competência do profissional de Enfermagem para punção de fístula arteriovenosa. [Internet] 2013 [acesso em 18 mai 2016]. Disponível: http://portal.coren-sp.gov.br/sites/default/files/parecer_coren_sp_2013_42.pdf.

13. Grothe C, Belasco AGS, Bittencourt ARC, Vianna LAC, Sesso RCC, Barbosa DA. Incidence of bloodstream infection among patients on hemodialysis by central venous catheter. Rev. Latino-Am Enfermagem. 2010; 18(1): 73-80.

14. Santana JCB, Fortes NM, Monteiro CLA, Carvalho IM, Leonardo LMU, de Albuquerque PG. Assistência de enfermagem em um serviço de terapia renal substitutiva: implicações no processo de cuidar. Enfermagem Revista. 2012; 15(2): 168-78. 